\title{
Vitamin D supplemental therapy can explore the interplay circuit between hypovitaminosis $D$, high serum IL-6 and pregnancy-associated morbidities
}

\begin{abstract}
Objectives: To evaluate the relation between baseline vitamin D (VD) serum levels and incidence and severity of pregnancy-associated morbidities (PAM).

Patients \& methods: The study included 386 pregnant women attended at the 6th gestational week and after full clinical and obstetric examination, gave fasting blood samples for estimation of fasting blood glucose (FBG) and ELISA estimation of serum VD, insulin and interleukin-6 (IL-6). Insulin resistance (IR) was determined according to homeostasis model assessment IR (HOMA-IR) score that was considered abnormal at score of $\geq 2$. Enrolled women were categorized according to at booking VD level as sufficient (Group A), insufficient (Group B) and deficient (Group C). Women of group $\mathrm{C}$ received daily oral VD (1000 IU) supplemental therapy (VD-ST) till delivery. Study outcomes included incidence of PAM and its relation to serum VD levels, and the effect of VD-ST on incidence and severity of PAM.

Results: Groups A-C included 86, 130 and 170 women, respectively and 263 women developed 424 PAM. PAM incidence and serum IL-6 levels were significantly higher in group B than other groups. PAM incidence and serum IL-6 levels showed negative significant correlations with serum VD levels. Moreover, PAM incidence showed positive significant correlation with serum IL-6 level. ROC curve analysis defined at booking serum VD as significant specific and serum IL-6 as significant sensitive predictors for PAM development. VD-ST allowed significant reduction of PAM with significant reduction of serum IL-6 and FBG levels and IR. Incidence of PAM was negatively correlated with receiving VD-ST that was found to be significant predictor for decreased PAM incidence.

Conclusion: Hypovitaminosis D and high IL-6 serum levels may underlie PAM development. VD-ST improved pregnancy outcome in women with VD deficiency to a level better than in women with insufficient VD levels; thus it is recommended as empirical therapy for pregnant women.
\end{abstract}

Keywords: hypovitaminosis D, interleukin-6, pregnancy-associated morbidities, VD-supplemental therapy
Volume 10 Issue 4 - 2019

Khaled Fathy Helal

Lecturer of Gynecology and Obstetrics Department, Faculty of Medicine, Zagazig University, Egypt

Correspondence: Khaled Fathy Helal, Lecturer of Gynecology and Obstetrics Department, Faculty of Medicine, Zagazig University, Zagazig, Egypt, Tel 0I55755675523,

Email olaharb2015@gmail.com

Received: July 04, 2019 | Published: July II, 2019

\section{Introduction}

Vitamin D is a prohormon with multiple functions that extend beyond the regulation of the intestinal calcium absorption. ${ }^{1}$ After being absorbed VD is bound to VD-binding protein and carried in blood stream to liver to undergo its first hydroxylation into 25-hydroxy VD. ${ }^{2}$ Hydroxylated VD will be further hydroxylated in kidneys to 1,25-dihydroxy VD; which is the biologically active form of $\mathrm{VD}^{3}$ through binding to its receptors present in skeletal and non-skeletal tissues. ${ }^{4}$ The placenta is an important source of VD and regulates its metabolism, and through the VD receptor in placental tissues, VD can regulate trophoblast function. ${ }^{5}$

Pregnant women form one of the high risk groups facing hypovitaminosis D (HVD), ${ }^{6}$ because the pregnant woman is the only source of VD for the fetus and the main sources of VD for pregnant women are sunlight, fortified dairy products, oily fish and dietary supplements. ${ }^{7}$ Thus, maternal nutrition may influence both the overall pregnancy outcome, immune system of the mother and fetus and the growth trajectory of the fetus and infant. ${ }^{8}$ Vitamin D deficiency is a common problem among Egyptian adolescent girls, for whom contributing factors include inadequate sun exposure possibly related to cultural/social factors, and insufficient dietary calcium. ${ }^{9}$ Due to; lack of sun exposure, presence of seasonal variations, dark skin pigmentation, clothing style, obesity and low intake of vitamin D. ${ }^{10}$

VD deficiency/insufficiency is prevalent among pregnant women ${ }^{11}$ and epidemiological studies had linked VD deficiency to adverse maternal and neonatal outcomes. ${ }^{5}$ Thus, screening for HVD in the first trimester of pregnancy is important to determine the necessary preventive action. ${ }^{6}$

Supplemental therapy (ST) during pregnancy is still a matter of discrepancy; ${ }^{12}$ concerning VD-ST, the current evidence base could not allow definite conclusions regarding the optimal maternal circulating concentration of $25 \mathrm{OH}-\mathrm{VD}$ during pregnancy, and how this might best be achieved. ${ }^{13}$ VD-ST during pregnancy was associated with increased its circulating levels, birth weight, and length, but was not associated with other outcomes. ${ }^{14}$ 
Gap of Knowledge: no previous studies discussed the relation between baseline VD serum levels and frequency and severity of PAM

Hypothesis: Hypovitaminosis D (HVD) is a global problem and mostly has an impact during pregnancy on both the mother and fetus. HVD may predispose or aggravate pregnancy-associated morbidities (PAM)

Objectives: The study aimed to evaluate the relation between baseline VD serum levels and frequency and severity of PAM.

Design: Prospective selective therapeutic trial.

Setting: Zagazig University Hospital, Zagazig, Egypt

Sample size: The study included 450 pregnant women; 39 patients were excluded and 411 women were evaluated, 25 women had escaped during follow-up and 386 women were enrolled in the study.

\section{Patients \& methods}

The study protocol was approved by the Institutional Review Board (IRB) of Faculty of Medicine Zagazig University and all enrolled women signed a written fully-informed consent to participate in the study. To exclude the seasonal variations of VD levels, the study intended to collect all women attended the antenatal care unit at Zagazig University Hospital at the $6^{\text {th }}$ week gestational age (6GW) since March $21^{\text {st }}$ till June $21^{\text {st }}$ each year since March 2014 were eligible for clinical evaluation and after preliminary evaluation were asked to re-attend the clinic fasting on the next day to give blood samples for investigations.

Preliminary examination included full clinical and obstetric history taking, determination of women's baseline anthropometric data and clinical data. Body mass index (BMI) was calculated according to the equation; $\mathrm{BMI}=$ weight $(\mathrm{kg}) /$ height $\left(\mathrm{m}^{2}\right)$ and patients were classified as underweight: $\mathrm{BMI}<18.5 \mathrm{~kg} / \mathrm{m}^{2}$, normal weight: $\mathrm{BMI}=18.5-24.9 \mathrm{~kg} /$ $\mathrm{m}^{2}$, overweight: $\mathrm{BMI}=25-29.9 \mathrm{~kg} / \mathrm{m}^{2}$ and obese: $\mathrm{BMI}=\geq 30 \mathrm{~kg} / \mathrm{m}^{2} .{ }^{13}$

Exclusion criteria included presence of multiple pregnancy, fetal congenital anomalies, current DM or essential hypertension, BMI $>35 \mathrm{~kg} / \mathrm{m}^{2}$, obesity-inducing endocrinopathy, evident manifestations of hypo-parathyroidism, thyrotocixosis, renal or hepatic diseases and women gave birth outside the hospital were also excluded from the study.

This study was conducted in compliance with all the applicable institutional ethical guidelines for the care, welfare and use of humans.

\section{Blood sampling and investigations}

Fasting blood samples were obtained from all women under complete aseptic conditions; blood samples obtained from the antecubital and were divided into three parts:

a. A part of blood sample was collected in a plane tube, till be clotted and then was centrifuged at $3000 \mathrm{rpm}$ for $10 \mathrm{~min}$. Serum was removed and collected in pyrogen-free Eppendorf tubes to be stored at $-70^{\circ} \mathrm{C}$ until ELISA assayed for serum levels of $25 \mathrm{OH}-$ VD, using an ELISA kit from Cayman Chemical, Ann Arbor, MI, USA,${ }^{4}$ insulin using ELISA kit from Quantikine R\&D systems Inc. Minneapolis MN USA ${ }^{14}$ and IL-6 using ELISA kit from Pelikine ${ }^{\mathrm{TM}}$ Inc., Concord, USA. ${ }^{15}$

b. Another part was put in a tube containing sodium fluoride $(2 \mathrm{mg}$ sodium fluoride $/ \mathrm{ml}$ blood) to prevent glycolysis. Plasma was separated by centrifugation and used for estimation of fasting blood glucose (FBG) by glucose oxidase method. ${ }^{16}$

c. A $3^{\text {rd }}$ part was used for routine laboratory investigations according to hospital protocol including complete blood count, serum urea and creatinine and serum liver function tests (results are not included).

\section{Evaluated parameters}

i. Vitamin D sufficiency status was defined, at booking time, according to $25-\mathrm{OHD}$ concentration as follows: $\geq 75 \mathrm{nmol} / \mathrm{L}$ sufficient level, $50-75 \mathrm{nmol} / \mathrm{L}$ insufficient level and $<50 \mathrm{nmol} / \mathrm{L}$ deficient level. ${ }^{17}$

ii. Insulin resistance (IR) was measured by homeostasis model assessment IR (HOMA-IR) score. The HOMA-IR score was calculated as fasting serum insulin $(\mu \mathrm{U} / \mathrm{ml}) \times$ [FBG $(\mathrm{mg} /$ $\mathrm{ml} / 18 \mathrm{18}) / 22.5^{18}$ was considered abnormal at HOMA-IR score of $\geq 2 .{ }^{19}$ IR parameters were estimated at both at booking time and the $36^{\text {th }} \mathrm{GW}$

iii. Gestational diabetes mellitus (GDM) was assesses at $36^{\text {th }} \mathrm{GW}$ using the $75 \mathrm{~g}$ Oral Glucose Tolerance Test and diagnosed according to guides of the International Association of Diabetes and Pregnancy Group for abnormal OGTT, as follows: FBG $\geq 92 \mathrm{mg} / \mathrm{dl}$, 1-h BG $\geq 180 \mathrm{mg} / \mathrm{dl}$ and $2-\mathrm{h} \mathrm{BG} \geq 153 \mathrm{mg} / \mathrm{dl}^{20}$

iv. Pre-eclampsia (PE) was diagnosed by development of gestational hypertension after the $12^{\text {th }}$ week GA in women who were normotensive at booking time with SBP $\geq 140 \mathrm{mmHg}$ and/or DBP $\geq 90 \mathrm{mmHg}$ on at least two occasions, 4 hours apart, and proteinuria defined as one dipstick measurement $\geq 2+$ on a voided random urine sample. ${ }^{21}$

v. Gestational anemia (GA) was defined as $\mathrm{Hb}$ conc. of $<11 \mathrm{gm} /$ $\mathrm{dl}$ either at booking time or at the $36^{\text {th }} \mathrm{GW} \cdot{ }^{22} \mathrm{Hb}$. deficit was calculated as the percentage of decrease in Hb. conc. at the $36^{\text {th }}$ $\mathrm{GW}$ in relation to that estimated at booking time.

\section{Patients' grouping}

Patients were categorized according to estimated baseline serum vitamin $\mathrm{D}$ into three groups

a) Patients with sufficient VD level were included as negative control group (Group A).

b) Patients with insufficient VD level were included as positive control group (Group B), were followed up and received no VD supplemental therapy (VD-ST).

c) Patients with deficient VD level were included as study group (Group C) and received VD-ST according to Grant et al. ${ }^{23}$ as a daily oral dose of 1000IU softgels to be taken with mail (Sunvite High Potency Vitamin D3 1000 IU; Puritan's Pride, Inc., Oakdale, NY, USA). VD-ST was started since study enrollment, after giving blood sample, till delivery.

\section{Study outcomes}

Primary outcome: the frequency of VD deficiency among studied women

Secondary outcomes: included 
A. Frequency of pregnancy-associated morbidities (PAM) among studied groups and its relation with estimated serum VD levels.

B. The effect of VD-ST on frequency and severity of PAM.

\section{Statistical analysis}

Obtained data were presented as mean $\pm \mathrm{SD}$, numbers and percentages. Results were analyzed using One-way ANOVA test, paired t-test and Chi-square test $\left(\mathrm{X}^{2}\right.$ test). Possible relationships were investigated using Pearson's linear regression. Sensitivity \& specificity of estimated parameters as predictors were evaluated using the receiver operating characteristic (ROC) curve analysis judged by

Table I At booking data of women of studied groups the area under the curve (AUC) compared versus the null hypothesis that $\mathrm{AUC}=0.05$. Statistical analysis was conducted using the IBM SPSS (Version 23, 2015) for Windows statistical package. P value $<0.05$ was considered statistically significant. ${ }^{24,25}$

\section{Results}

The study included 386 women. At booking time, estimated serum VD defined 86 women having sufficient VD level (Group A), 130 women had insufficient serum VD (Group B) and 170 women had deficient serum VD (Group C) (Figure 1). Apart from serum VD, other enrolment data of studied women showed non-significant $(\mathrm{p}>0.05)$ difference as shown in Table 1.

\begin{tabular}{|c|c|c|c|c|c|}
\hline \multicolumn{2}{|l|}{ Variable } & Group A & Group B & Group C & $P$ value \\
\hline \multicolumn{2}{|l|}{ Number (\%) } & $86(22.3 \%)$ & $130(33.7 \%)$ & $170(44 \%)$ & 0 \\
\hline \multicolumn{2}{|l|}{ Age (years) } & $27.3 \pm 4.7$ & $28.1 \pm 4.3$ & $27.4 \pm 3.9$ & 0.224 \\
\hline \multicolumn{2}{|l|}{ Weight (kg) } & $80.5 \pm 8.5$ & $77.3 \pm 10$ & $78.8 \pm 9.4$ & 0.054 \\
\hline \multicolumn{2}{|l|}{ Height (cm) } & $170.4 \pm 3.7$ & $169.2 \pm 3$ & $169.9 \pm 3.5$ & 0.148 \\
\hline \multicolumn{2}{|l|}{ BMI (kg/m2) } & $27.7 \pm 3.1$ & $27.2 \pm 3.6$ & $27.3 \pm 3.2$ & 0.089 \\
\hline \multicolumn{2}{|l|}{ Gravidity } & $2.4 \pm 1.1$ & $2.2 \pm 0.8$ & $2.2 \pm 0.7$ & 0.258 \\
\hline \multicolumn{2}{|l|}{ Parity } & $1.3 \pm 1$ & $1.1 \pm 0.9$ & $1.3 \pm 0.8$ & 0.346 \\
\hline \multicolumn{2}{|c|}{ Systolic blood pressure (mmHg) } & $118.5 \pm 6.1$ & $120.1 \pm 8.3$ & $120.8 \pm 7$ & 0.693 \\
\hline \multicolumn{2}{|c|}{ Diastolic blood pressure $(\mathrm{mmHg})$} & $72 \pm 3$ & $73.4 \pm 5.9$ & $75.4 \pm 7.8$ & 0.476 \\
\hline \multicolumn{2}{|c|}{ Serum VD (nmol/L) } & $77.7 \pm 2.1$ & $58.6 \pm 5.2$ & $31.7 \pm 9.3$ & 0.0001 \\
\hline \multicolumn{2}{|c|}{ Hb conc. (gm) } & $11.7 \pm 0.8$ & $11.6 \pm 0.8$ & $11.7 \pm 0.9$ & 0.686 \\
\hline \multirow[t]{3}{*}{ Lab data } & FBG (mg/dl) & $116.8 \pm 7.7$ & $116 \pm 5.9$ & $114.8 \pm 7.1$ & 0.079 \\
\hline & $\mathrm{FSI}(\mu \mathrm{U} / \mathrm{ml})$ & $5 \pm 1.23$ & $5.26 \pm 1.23$ & $5.22 \pm I .1$ & 0.242 \\
\hline & HOMA score & $1.44 \pm 0.35$ & $1.5 \pm 0.35$ & $|.48 \pm 0.3|$ & 0.396 \\
\hline
\end{tabular}

Throughout observation period 263 women developed 424 PAM for a frequency of 1.61 PAM/woman. Regarding the frequency among studied groups; 50 (58.1\%), $104(77.7 \%)$ and 109 (64.1\%) women developed PAM in groups A-C, respectively (Figure 1). There was significant variance $(\mathrm{p}=0.001)$ among studied groups regarding the incidence of PAM among studied groups, but in favor of group A. The incidence of PAM among women of group B was significantly $(p=0.0005)$ higher than groups $A$ and $C(p=0.0005 \& 0.0027$, respectively), but the difference was non-significantly $(\mathrm{p}=0.352)$ higher in group $\mathrm{C}$ than in group $\mathrm{A}$.

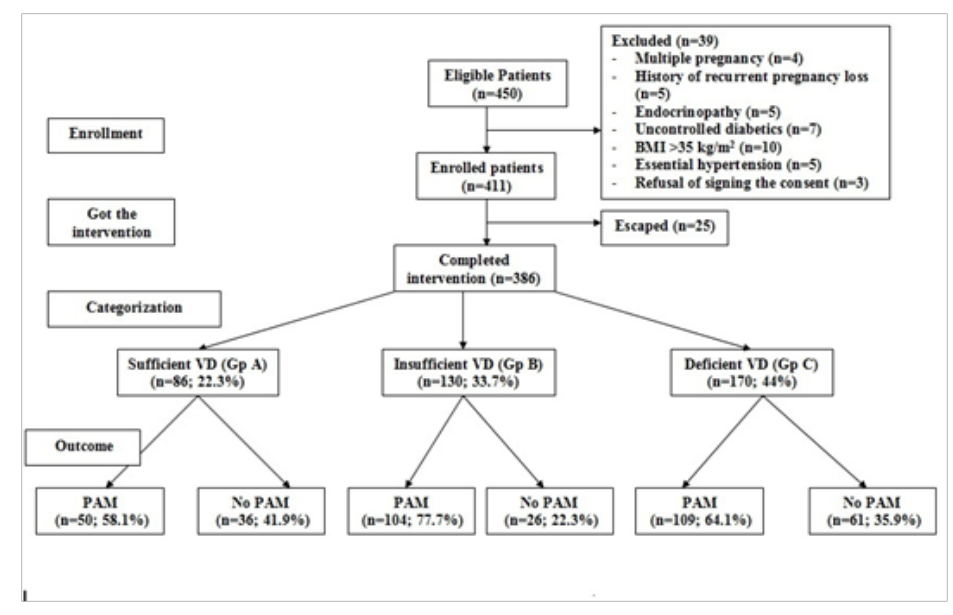

Figure I Flow chart of the study. 
Hemoglobin conc. determined at the $36^{\text {th }} \mathrm{GW}$ and calculated deficit than at booking concentration showed significant $(\mathrm{p}=0.0001$ \& 0.0089 , respectively) difference between studied groups, but in favor of Group A. Development of gestational anemia (GA) was the most frequently recorded PAM; where 194 women developed GA. Differentially, 39, 75 and 80 women in groups A-C, respectively developed GA with non-significant $(\mathrm{p}=0.111)$ difference between the studied groups, but also in favor of group A (Table 2).

At booking time, no patient had IR, while at the $36^{\text {th }} \mathrm{GW}, 112$ patients developed IR (29\%); 14 in group A and 51 and 47 in groups $\mathrm{B}$ and $\mathrm{C}$, respectively. The frequency of women developed IR at the $36^{\text {th }} \mathrm{GW}$ showed significant $(\mathrm{p}=0.012)$ variance among studied women with significantly higher frequency among women of group B compared to groups $\mathrm{A}(\mathrm{p}=0.0003)$ and $\mathrm{C}(\mathrm{p}=0.034)$ and nonTable 2 At $36^{\text {th }} \mathrm{GW}$ laboratory findings and IR data of women of studied groups significantly ( $\mathrm{p}=0.063$ ) higher IR women in group $\mathrm{C}$ versus group $\mathrm{A}$. Among women developed IR, 59 women (16\%) progressed to GDM, despite of the significantly higher frequency of IR in women with HVD (groups B and C), the frequency of women developed GDM was non-significantly higher among women had HVD compared to those had sufficient VD levels (Table 2).

Interestingly, all women showed significantly higher serum IL-6 compared to the standard control levels, as documented by manufacturer, with significant $(\mathrm{p}=0.0001)$ variance among studied groups. Moreover, women of group B had significantly higher serum IL-6 compared to those of groups A and $C(p=0.00006 \& 0.0012$, respectively) with non-significantly ( $\mathrm{p}=0.133$ ) higher levels in women of group $\mathrm{C}$ than women of group A (Table 2) (Figure 2).

\begin{tabular}{|c|c|c|c|c|c|c|}
\hline Variable & & & Group A & Group B & Group C & $P$ value \\
\hline & Conc. (gm) & & $\mathrm{II} \pm 0.77$ & $10.64 \pm 0.72$ & $10.68 \pm 0.72$ & 0.0001 \\
\hline \multirow[t]{4}{*}{ Hb. data } & Deficit & & $5.95 \pm 1.5$ & $8.53 \pm 3.18$ & $8.72 \pm 3.72$ & 0.0089 \\
\hline & GA & & 39 (45.3\%) & 75 (57.7\%) & 80 (47.1\%) & 0.111 \\
\hline & FBG & & $122.8 \pm 15.9$ & $130.1 \pm 18.6 *$ & $123.9 \pm 17.9$ & 0.0029 \\
\hline & FSI & & $4.9 \pm 1.2$ & $5.4 \pm 1.2$ & $5.59 \pm 1$ & 0.00006 \\
\hline \multirow[t]{3}{*}{ IR data } & & $<2$ & 74 (83.7\%) & 79 (60.8\%) & $123(72.4 \%)$ & 0.012 \\
\hline & HOMA score & $\geq 2$ & $14(16.3 \%)$ & $5 \mathrm{I}(39.2 \%)$ & 47 (27.6\%) & \\
\hline & & Mean & $1.5 \pm 0.5$ & $1.77 \pm 0.54$ & $1.72 \pm 0.44$ & 0.0009 \\
\hline IL-6 (ng/ml) & & & $35.2 \pm 16.4$ & $44.4 \pm 16$ & $41.8 \pm 14.4$ & 0.0001 \\
\hline
\end{tabular}

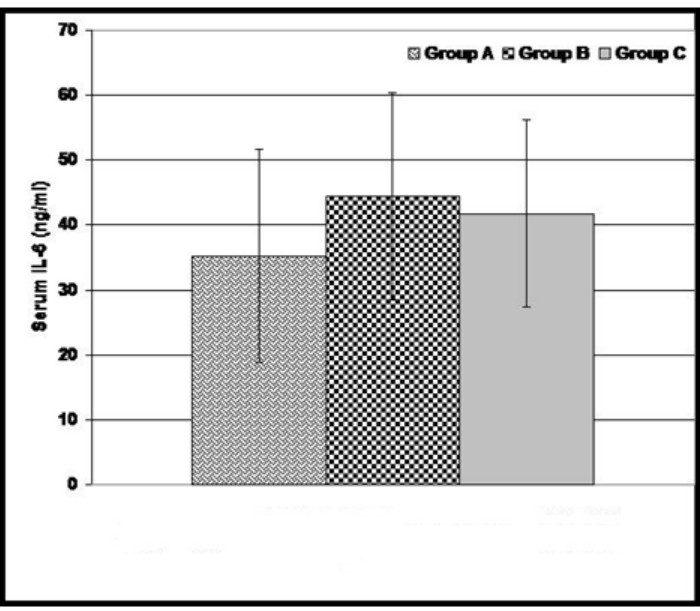

Figure 2 Serum levels of IL-6estimated in women according to VD sufficiency levels.

Thirty-four women (8.8\%) developed pre-eclampsia; 5, 14 and 15 women in groups $\mathrm{A}-\mathrm{C}$, respectively with non-significant $(\mathrm{p}=0.453)$ variance among women in the three groups, but in favor of group A. Twenty-four women had preterm labor with non-significant $(p=0.449)$ difference between the three groups, but in favor of group A. Total incidence of PAM, frequency of women developed PE, serum IL-6 levels, frequency of women had preterm labor and HOMA-IR score showed negative significant correlation with at booking serum $\mathrm{VD}$, in decreasing order of frequency, while $\mathrm{Hb}$. Conc. at $36^{\text {th }} \mathrm{GW}$ showed positive significant correlation with at booking serum VD. On the other hand, total incidence of PAM, HOMA-IR, GDM, PE and preterm labor showed positive significant correlation with at $36^{\text {th }}$ GW serum IL-6 level, while at $36^{\text {th }} \mathrm{GW} \mathrm{Hb}$. Conc. was negatively correlated with serum IL-6 (Table 3). ROC curve analysis defined at booking serum VD as significant $(\mathrm{p}=0.02)$ specific predictor with $\mathrm{AUC}=0.573( \pm 0.032 ; 95 \% \mathrm{CI}=0.510-0.636)$ and serum IL-6 as significant $(\mathrm{p}=0.0007)$ sensitive predictor with $\mathrm{AUC}=0.385( \pm 0.03$; 95\% CI $=0.327-0.443$ ) for development of PAM (Figure 3). Women of group C who received VD-ST had improved pregnancy outcome as manifested by the significantly lower incidence of PAM compared to women of group B. The reported beneficial effect of VD-ST was evidenced by the significant reduction of serum IL-6 ( $p=0.0012)$, FBG 
level $(\mathrm{p}=0.004)$ and IR $(\mathrm{p}=0.034)$. Moreover, the incidence of PAM was negatively $(r=-0.142, p=0.014)$ correlated with receiving VD-ST and maintenance on VD-ST by women had deficient VD was found

Table 3 Correlation between at booking serum VD and at $36^{\text {th }}$ GW serum IL- 6 with incidence rate of PAM

\begin{tabular}{lllll}
\hline \multirow{2}{*}{ Variables } & \multicolumn{2}{c}{ Serum VD } & \multicolumn{2}{l}{ Serum IL-6 } \\
\cline { 2 - 5 } & "r" & $\mathbf{p}$ & "r" & $\mathbf{p}$ \\
\hline Serum IL-6 & -0.133 & 0.009 & - & - \\
Total frequency of PAM & -0.162 & 0.001 & 0.327 & 0.0003 \\
At $36^{\text {th }}$ GW Hb. Conc. & 0.133 & 0.009 & -0.183 & 0.0008 \\
At $36^{\text {th }}$ GW HOMA-IR score & -0.122 & 0.017 & 0.27 & 0.0006 \\
GDM & -0.082 & 0.108 & 0.193 & 0.0009 \\
Frequency of PE & -0.14 & 0.006 & 0.134 & 0.008 \\
Frequency of preterm labor & -0.126 & 0.013 & 0.116 & 0.023 \\
\hline
\end{tabular}

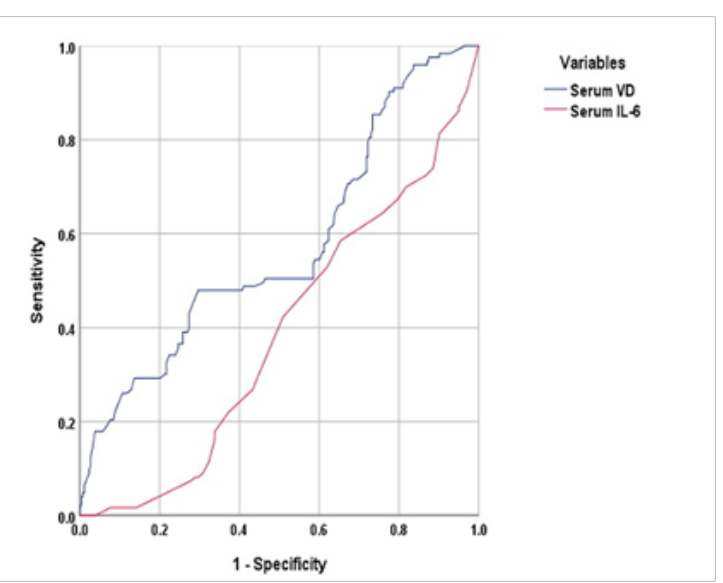

Figure 3 ROC curve for diagnostic validity of at booking serum VD and at 36th GW serum IL-6 for PAM development.

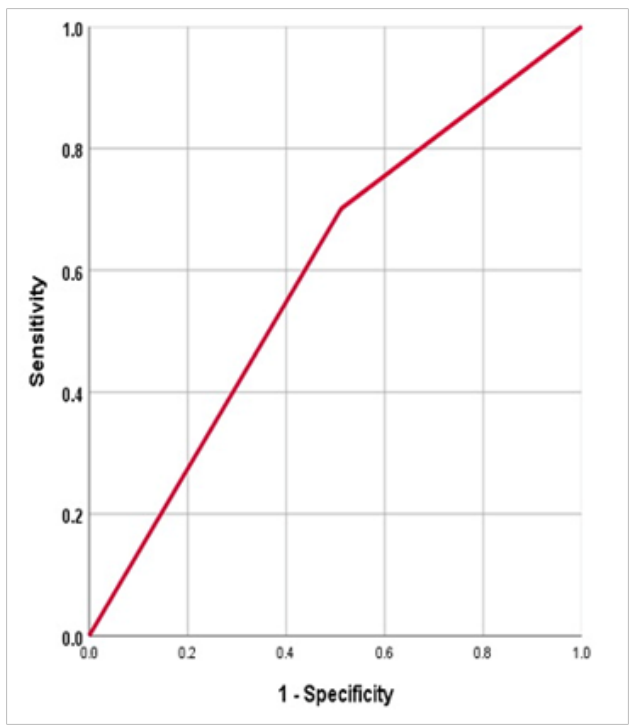

Figure 4 ROC curve for diagnostic validity of receiving VD-ST at booking time as predictor for sparing PAM development. 
Data are presented as numbers. Percentages, mean $\pm \mathrm{SD}$; BMI: Body mass index; VD: Vitamin D; Hb. conc.: Hemoglobin concentration; IR: Insulin resistance; FBG: Fasting blood glucose; FSI: Fasting serum insulin; HOMA: homeostasis model assessment IR; $p$ value indicates statistical variance between groups and $p>0.05$ indicates non-significant difference

\section{Discussion}

The reported incidence of HVD among studied pregnant women was $77.7 \%$, considering timing of sample at the $6^{\text {th }} \mathrm{GW}$, so HVD could not be attributed to pregnancy and was considered as preconception condition that most probably attributed to environmental and nutritional factors. In line with these data, Karras et al. ${ }^{12}$ detected a prevalence of VD insufficiency and deficiency among pregnant women in Mediterranean region in ranges of 9.3-41.4\% and 22.7$90.3 \%$, respectively. Thereafter, Naseh et al. ${ }^{11}$ reported VD deficiency in $27 \%$ and insufficiency in $73 \%$ of their sample of Iranian pregnant women. Moreover, Chakhtoura et al. ${ }^{26}$ found the prevalence of HVD, defined as a 25 -hydroxyvitamin $\mathrm{D}$ level $<50 \mathrm{nmol} / \mathrm{L}$, ranged between 44 and $96 \%$ in adults and $54-90 \%$ in pregnant women.

During the study duration, 263 women developed 424 PAM; 1.61 $\mathrm{PAM} /$ woman; differentially, at the $36^{\text {th }} \mathrm{GW}, 112$ women developed IR and 59 of them progressed to GDM, 34 women developed PE and 24 women had preterm labor. There was significant variance among studied women categorized according to at booking serum VD levels regarding total and differential frequency of PAM.

Multiple clinical studies detected similar associations where Michalski et al. ${ }^{27}$ found low VD status may be linked to reduced $\mathrm{Hb}$. Conc. in pregnant women and Yuan et al. ${ }^{28}$ reported that deficient maternal serum VD may be a risk factor for GA and it should be monitored especially for the high-risk pregnant women. Also, Amegah et al. ${ }^{29}$ detected increased risk of preterm birth with serum VD levels $<75 \mathrm{nmol} / 1$ with an inverse dose-response relation.

Recently, Windrim et al..$^{30}$ documented that VD deficiency during pregnancy has maternal and fetal implications, with increased risk of developing GDM, PE, preterm birth and small for gestational age birth weight. Also, Hong-Bi et al. ${ }^{31}$ detected negative correlation between VD levels and adverse pregnancy outcomes. Moreover, Serrano et al..$^{32}$ reported increased probability of having PE among women with VD deficiency, relative to controls.

Serum IL-6 showed significant variance among studied groups with a positive significant correlation with incidence of PAM, HOMA-IR, GDM, PE and preterm labor, while showed negative significant correlation with at $36^{\text {th }} \mathrm{GW} \mathrm{Hb}$. Conc. and deficit. These findings go in hand with Oztas et al. ${ }^{33}$ who documented that higher $1^{\text {st }}$ trimester IL-6 levels can independently predict adverse pregnancy outcomes. Also, Li et al. ${ }^{34}$ suggested that high serum TNF- $\alpha$ and IL-6 levels are associated with pregnancy-induced hypertension and might be potential predictors for its prognosis. Moreover, Zhang et al. ${ }^{35}$ found IL-6, IL-8, and IL-6/IL-10, serious inflammation and disordered lipid and glucose metabolisms were associated with GDM in women. Recently, Krasnyi et al. ${ }^{36}$ documented that PE develops upon increased macrophage activity with subsequent increased serum levels of its factors; IL-10 and IL-6 leading to destruction of the placental trophoblastic cells.

Women with deficient serum VD who received VD-ST had improved outcome manifested as significantly lower incidence of PAM with significant reduction of serum IL-6 and FBG levels, and
HOMA-IR score. VD-ST was found to specific significant $(\mathrm{p}=0.010)$ predictor for decreased incidence of PAM with $\mathrm{AUC}=0.595$. In line with the beneficial effect of VD-ST on pregnancy outcome, Naseh et al. ${ }^{11}$ suggested promoting consumption of VD-fortified foods and supplements among pregnant women to improve maternal and neonatal outcomes. Also, Krieger et al. ${ }^{37}$ suggested that VD-ST during pregnancy should receive more attention in clinical practice as it was the most actionable determinant of VD status at the $3^{\text {rd }}$ trimester and in neonatal cord blood and Wheeler et al. ${ }^{38}$ recommended $1^{\text {st }}$ trimester maternal VD screening and targeted supplementation for those "at risk".

The dose of VD-ST was adjusted according to Grant et al. ${ }^{25}$ in the form of 1000IU softgels to be taken orally with mail starting since $1^{\text {st }}$ antenatal visit till delivery. Similarly, Cooper et al. ${ }^{39}$ found supplementation of women with cholecalciferol 1000IU/day during pregnancy is safe and sufficient to ensure repletion of VD stores in pregnant women and Yilmaz et al. ${ }^{40}$ documented that VD-ST with $1200 \mathrm{IU} /$ day from the $12^{\text {th }} \mathrm{GW}$ can prevent neonatal VD deficiency. Also, Chakhtoura et al. ${ }^{41}$ reported that in pregnant women VD dose of 1000-2000IU daily may be necessary to allow for the majority of this population to have serum VD level of $50 \mathrm{nmol} / \mathrm{L}$.

\section{Conclusion}

Pregnancy is stressful period during women's life and is associated with high incidence of morbidities. HVD and high IL-6 serum levels form a closed circuit that may underlie development of pregnancyassociated morbidities. VD-ST is a promising therapeutic option as it improved pregnancy outcome in women with VD deficiency to a level better than in women with insufficient VD levels; thus it is recommended to be applied in a similar dose as empirical therapy for all pregnant women.

Authors' contribution: both authors share in data collection, statistical analysis, paper design, writing and reviewing the paper before submission.

Limitations of the work: Absent of a long period of evaluation of patients and absence of data about VD levels before pregnancy.

\section{Acknowledgments}

None.

\section{Conflicts of interest}

Authors declared no conflicts of interests or financial affairs.

\section{References}

1. Muscogiuri G, Altieri B, Annweiler C, et al. Vitamin D and chronic diseases: the current state of the art. Arch Toxicol. 2017;91(1):97-107.

2. Lapillonne A. Vitamin D, deficiency during pregnancy may impair maternal and fetal outcomes. Med Hypotheses. 2010;74(1):71-75.

3. Cho GJ, Hong SC, Oh MJ, et al. Vitamin D deficiency in gestationa diabetes mellitus and the role of the placenta. Am J Obstet Gynecol. 2013;209(6):560.e1-e8.

4. Teegarden D, Donkin SS. Vitamin D: emerging new roles in insulin sensitivity. Nutr Res Rev. 2009; 22(1):82-92.

5. Nguyen TP, Yong HE, Chollangi T, et al. Placental vitamin D receptor expression is decreased in human idiopathic fetal growth restriction. $J \mathrm{Mol}$ Med (Berl). 2015;93(7):795-805. 
6. Bukhary NBI, Isa ZM, Shamsuddin $\mathrm{K}$, et al. Risk factors for antenatal hypovitaminosis $\mathrm{D}$ in an urban district in Malaysia. BMC Pregnancy Childbirth. 2016;16(1):156.

7. Dovnik A, Mujezinović F. The association of vitamin D levels with common pregnancy complications. Nutrients. 2018;10(7).

8. Berti C, Agostoni C, Davanzo R, et al. Early-life nutritional exposures and lifelong health: immediate and long-lasting impacts of probiotics, vitamin D, and breastfeeding. Nutr Rev. 2017;75(2):83-97.

9. Amr N, Hamid A, Sheta M, et al. Vitamin D status in healthy Egyptian adolescent girls. Georgian Med News. 2012;(210):65-71.

10. El Rassi AR, Fuleihan GE. Hypovitaminosis D in developing countries - prevalence, risk factors and outcomes. Nat Rev Endocrinol. 2010;6(10):550-561.

11. Naseh A, Ashrafzadeh S, Rassi S. Prevalence of vitamin D deficiency in pregnant mothers in Tehran and investigating its association with serum glucose and insulin. J Matern Fetal Neonatal Med. 2018;31(17):23122318 .

12. Karras SN, Anagnostis P, Paschou SA, et al. Vitamin D status during pregnancy: time for a more unified approach beyond borders? Eur J Clin Nutr. 2015;69(8):874-877.

13. Harvey NC, Holroyd C, Ntani G, et al. Vitamin D supplementation in pregnancy: a systematic review. Health Technol Assess. 2014;18(45):1190.

14. Wagner CL, Hollis BW, Kotsa K, et al. Vitamin D administration during pregnancy as prevention for pregnancy, neonatal and postnatal complications. Rev Endocr Metab Disord. 2017;18(3):307-322.

15. Moyer VA, U.S. Preventive Services Task Force. Screening for and management of obesity in adults: U.S. Preventive Services Task Force recommendation statement. Ann Intern Med. 2012;157(5):373-378.

16. Andersen L, Dinesen B, Jørgensen PN, et al. Enzyme immunoassay for intact human insulin in serum or plasma. Clin Chem. 1993;39(4):578-582.

17. Gaines-Das RE, Poole S. The international standard for interleukin-6 evaluation in an international collaborative study. J Immunol Methods. 1993;160(2):147-153.

18. Tinder P. Determination of Glucose in Blood Using Glucose Oxidase with an Alternative Oxygen Acceptor. Ann Clin Biochem. 1969;6(1):24-27.

19. Stroud ML, Stilgoe S, Stott VE, et al. Vitamin D - a review. Aust Fam Physician. 2008;37(12):1002-1005.

20. Matthews DR, Hosker J, Rudenski A, et al. Homeostasis model assessment: insulin resistance and beta-cell function from fasting plasma glucose and insulin concentrations in man. Diabetologia. 1985;28(7):412-419.

21. Ascaso JF, Romero P, Real JT, et al. Insulin resistance quantification by fasting insulin plasma values and HOMA index in a non-diabetic population. Med Clin (Barc). 2001;117(14):530-533.

22. International Association of Diabetes and Pregnancy Study Groups Consensus Panel. International association of diabetes and pregnancy study groups (IADPSG) recommendations on the diagnosis and classification of hyperglycemia in pregnancy. Diabetes Care. 2010;33(3):676-682.

23. Gifford RW, August PA, Cunningham FG. Report of the national high blood pressure working group on research on hypertension in pregnancy. Am J Obstet Gynecol. 2000;183:S1-S22.

24. Api O, Breyman C, Cetiner M, et al. Diagnosis and treatment of iron deficiency anemia during pregnancy and the postpartum period: Iron deficiency anemia working group consensus report. Turk J Obstet Gynecol. 2015;12(3):173-181.
25. Grant CC, Stewart AW, Scragg R, et al. Vitamin D during pregnancy and infancy and infant serum 25-hydroxyvitamin D concentration. Pediatrics. 2014;133(1):e143-e153.

26. Chakhtoura M, Rahme M, Chamoun N, et al. Vitamin D in the Middle East and North Africa. Bone Rep. 2018;8:135-146.

27. Michalski ES, Nguyen PH, Gonzalez-Casanova I, et al. Serum 25-hydroxyvitamin D but not dietary vitamin D intake is associated with hemoglobin in women of reproductive age in rural northern Vietnam. $J$ Clin Transl Endocrinol. 2017;8:41-48.

28. Yuan Y, Cai Z, Dai Y, et al. Association of maternal serum 25-hydroxyvitamin D concentrations with risk of gestational anemia. Cell Physiol Biochem. 2017;43(4):1526-1532.

29. Amegah AK, Klevor MK, Wagner CL. Maternal vitamin D insufficiency and risk of adverse pregnancy and birth outcomes: A systematic review and meta-analysis of longitudinal studies. PLoS One. 2017;12(3):e173605.

30. Windrim CM, Crosby DA, Mitchell K, et al. Vitamin D supplementation in pregnancy-a survey of compliance with recommendations. Ir $\mathrm{J}$ Med Sci. 2018;187(3):709-712.

31. Hong-Bi S, Yin $\mathrm{X}$, Xiaowu $\mathrm{Y}$, et al. High prevalence of vitamin D deficiency in pregnant women and its relationship with adverse pregnancy outcomes in Guizhou, China. J Int Med Res. 2018;46(11):4500-4505.

32. Serrano NC, Guío E, Quintero-Lesmes DC, et al. Vitamin D deficiency and pre-eclampsia in Colombia: PREVitD study. Pregnancy Hypertens. 2018;14:240-244.

33. Oztas E, Erkenekli K, Ozler S, et al. First trimester interleukin-6 levels help to predict adverse pregnancy outcomes in both thyroid autoantibody positive and negative patients. J Obstet Gynaecol Res. 2015;41(11):1700 1707.

34. Li Y, Wang Y, Ding X, et al. Serum levels of TNF- $\alpha$ and IL-6 are associated with pregnancy-induced hypertension. Reprod Sci. 2016;23(10):14021408 .

35. Zhang J, Chi H, Xiao H, et al. Interleukin 6 (IL-6) and tumor necrosis factor $\alpha$ (TNF- $\alpha)$ single nucleotide polymorphisms (SNPs), inflammation and metabolism in gestational diabetes mellitus in inner Mongolia. Med Sci Monit. 2017;23:4149-4157.

36. Krasnyi AM, Gracheva MI, Sadekova AA, et al. Complex analysis of total and fetal DNA and cytokines in blood plasma of pregnant women with preeclampsia. Bull Exp Biol Med. 2018;164(6):721-725.

37. Krieger JP, Cabaset S, Canonica C, et al. Prevalence and determinants of vitamin $\mathrm{D}$ deficiency in the third trimester of pregnancy: a multicentre study in Switzerland. Br J Nutr. 2018;119(3):299-309.

38. Wheeler BJ, Taylor BJ, de Lange M, et al. A longitudinal study of 25-hydroxy vitamin $\mathrm{D}$ and parathyroid hormone status throughout pregnancy and exclusive lactation in New Zealand mothers and their infants at $45^{\circ} \mathrm{S}$. Nutrients. $2018 ; 10(1)$.

39. Cooper C, Harvey NC, Bishop NJ, et al. Maternal gestational vitamin D supplementation and offspring bone health (MAVIDOS): a multicentre, double-blind, randomised placebo-controlled trial. Lancet Diabetes Endocrinol. 2016;4(5):393-402.

40. Yılmaz B, Aygün C, Çetinoğlu E. Vitamin D levels in newborns and association with neonatal hypocalcemia. J Matern Fetal Neonatal Med. 2017;31(14):1889-1893.

41. Chakhtoura M, El Ghandour S, Shawwa K, et al. Vitamin D replacement in children, adolescents and pregnant women in the Middle East and North Africa: A systematic review and meta-analysis of randomized controlled trials. Metabolism. 2017;70:160-176. 